\title{
Zur Aetiologie und Therapie, speziell Balneotherapie der Psoriasis vulgaris.
}

Von Prof. H. Köbner in Berlin-Charlottenburg.

Der alte Satz, je mehr Arzneimittel gegen eine Krankheit empfohlen werden, desto weniger ist sie heilbar, gilt auch für die ätiologischen Hypothesen, welche bezüglich der Psoriasis aufgestellt worden sind. Entspräche ihrer Zahl die Kenntnis der wirklichen Ursachen dieser Krankheit, so wäre das Dunkel, welches noch heutzutage über diese herrscht, längst gelichtet, und auch die Therapie hätte daraus Nutzen gezogen.

Streifen wir kurz die ätiologischen Irrtümer von drei dermatologischen „Autoritäten“, welche sämtlich auf der Annahme hypothetischer Dyskrasien beruhen, so begegnen wir zuerst der Erasmus Wilsons ${ }^{1}$ ), Psoriasis beruhe auf einem Atavismus: von den Großeltern ererbter Syphilis, sodann Poors: ${ }^{2}$ ) - auf Malaria. (Meine privaten Ermittelungen bei Aerzten Ungarns ergaben jedoch, daß Psoriasis in Malariagegenden dieses Landes nicht häufiger ist als in malariafreien.) $\mathrm{Bazin}^{3}$ ) glaubte, ihre Ursache an der Farbe der Effloreszenzen unterscheiden zu können, und verordnete demgemäß bei der supponierten „Psoriasis arthritique" innerlich Alkalien, bei der "Psoriasis dartreuse“ Arsen. Er übersah aber, daß die lokalen Heilerfolge durch das stets gleichzeitig applizierte 01 . cadin. bewirkt worden waren.

Wie mit einem Schlage änderte sich die ätiologische Auffassung durch das Hinzutreten einer neuen Möglichkeit: der durch meine Ritzungsversuche an intakten, von Psoriasisplaques ganz entfernten Hautstellen von Psoriatikern bewirkten Entstehung neuer Plaques ${ }^{4}$ ), und zwar nicht etwa ausgehend vom Rande alter,

1) Wils on, Treatise on skin diseases. London 1867 und 1875. - 2) Poor, Prager Vierteljahrsschrift für Heilkunde 1878. - 3) B a z i n, Leçons sur les affection arthritiques et dartreuses. Paris 1861 - 4) $\mathrm{K}$ ob b ner, Aetiologie der Psoriasis. Jahresbericht der Schlesischen Gesellschaft für vaterländische Kultur 1872. Referiert im Archiv für Dermatologie und Syphilis 1876, S, 559 . 
seit Jahren stationärer, sondern frisch entstandener. Diese Entwicklung geschah so rasch und unmittelbar, daß schon wenige Tage nach diesem Trauma der Ausgangspunkt der teils isoliert bleibenden und nur an der Peripherie ein wenig wachsenden, teils durch Konfluenz gyrierte Formen annehmenden Figuren kaum zu erkennen war. Ich habe durch Skarifikationen der Haut solcher Kranken an der Vorderfläche des Stammes, und zwar symmetrisch von einer beide Mammillae verbindenden Querlinie abwärts bis zu den Inguinalgegenden mit dem schröpfschnäpperähnlichen, sogenannten Baunscheidtschen Instrument, vollkommen symmetrische, wie die Metallknöpfe einer Uniform angeordnete Plaques erzielt. Ebensolche erzielte ich nach beliebig, sei es rechtwinklig oder ellipsoid eingeritzten Figuren, deren fortlaufende Entwicklungsgeschichte ich durch A. Weyl') abbilden lieh.

Durch diese, einen klinisch mir zugewiesenen Fall, betreffend einen kräftigen 26jährigen Kürassier, angeregte Idee wurden die altbekannten, aber noch von niemandem zu erklären versuchten kardinalen Eigenschaften der Psoriasis: ihre Prädilektionsstellen (über Röhrenknochen und dem Schädel - also wo die Haut keinem äuferen Druck ausweichen kann -), die meistens zu beobachtende Symmetrie an denselben Druck- oder symmetrischen chemischen Reizstellen, z. B. den schon von älteren Beobachtern (Ray er, Hebra) aufgelegten Vesikatoren, und die Nutzlosigkeit der lokalen Therapie der Plaques für die gesamte Haut aufgehellt.

Diese letzte Bemerkung zielte hauptsächlich gegen $F$. und H. Hebra, welche ich die Schuppen mit dem scharfen Löffel hatte abreißen sehen; ich wollte die Nutzlosigkeit dieses Verfahrens gegen Rezidive betonen.

Bei jenem Kürassier, der angab, früher ganz ausschlagsfrei gewesen zu sein, hatten der oberflächliche Biß (angeblich nur Ritzung, ohne Blutung) eines jungen Remontepferdes an der Beugeseite des linken Vorderarmes Exkoriationen an beiden inneren Condylis der Oberschenkel, welche Patient beim Reiten ohne Sattel fest anzudrücken pflegte, und an der frischen Schnittnarbe eines konsekutiven linksseitigen Bubo intensiv beschuppte Plaques bewirkt. Am meisten hatten nachher Wannenbäder mit S enf zur Verallgemeinerung der Psoriasis beigetragen.

W. Scholz sah Regelmäßigkeit der „Abheilung von zwei Psoriasisgürteln" am Rücken von oben nach unten, zuerst eines solchen über den Spinae scapulae - mit Hinterlassung tief brauner Pigmentierung - , nachher eines zweiten in der Lumbalgegend nach subkutanen Arseninjektionen selbst in entferntesten Körpergegenden, z. B. Oberschenkel und -arme. Er leitete daraus seine "bioskopische Methode" zur Erkennung von Spuren Arsens in den Schuppen und Haaren durch Aussaat einer bestimmten Penicilliumspezies auf festen und flüssigen Nährböden ab.

Durch diese Wahrnehmungen wurde vollends die Hypothese eines parasitären Ursprunges widerlegt.

Durch Wechselmann ließ ich in der Berliner medizinischen Gesellschaft an einem Hund und einem $1 \frac{1}{2}$ jähriges Mädchen, welchem jener oft zum Spielen ins Bett gereicht worden war, Dermato- und Trichomykosisherde demonstrieren. Bei dem Hunde, der, ein vorzüglicher Rattenfänger, in einem an Ratten reichen Stall eingesperrt gewesen war, zeigten sich vorwiegend auf der Schnauze, der vorderen Bauch- und der Innenseite der Extremitäten ebensolche kahle Flecken, wie bei dem Kinde an den entblößt getragenen Armen und dem oberen Teil der Brustgegend. Auch impfte ich von auf das feinste zerriebenen Psoriasisschuppen auf die frisch skarifizierte Armhaut eines psoriatischen Assistenten ohne Erfolg.

Von schwächenden, neue Eruptionen begünstigenden Faktoren kommt zunächst in Betracht die Laktation.

Eine Patientin Tommasolis bekam im ersten Wochenbett den ersten Ausbruch von Psoriasis am behaarten Kopf. Während der folgenden Gravjdität ging diese völlig zurück, erschien aber wieder während der Laktation! Und dieser Turnus wieder holte sich bei 11 Graviditäten.

Pel berichtet, dal ein 50 jähriger Arbeiter 30 Jahre lang in jedem Spätsommer zusammen mit - einer Psoriasis universalis an den Symptomen einer Lateralsklerose erkrankte und in jedem Frühahr gleichzeitig sowohl von den Symptomen dieser als auch von jener genas.

Dasselbe sah ich bei einer 36 jährigen Frau, welche ich als zwölfjähriges Mädchen an Psoriasis behandelt hatte und welche, meiner ihrer Mutter mitgeteilten Voraussage gemäß, nach jedem Wochenbett, beziehungsweise Laktation ihrer fünf Töchter neue Ausbrüche bekam.

1) A. Weyl, Abschnitt "Psoriasis" in v. Ziemssens Handbuch der Hautkrankheiten
Bei einem mit Tinct. jodi über einem entzündeten Kniegelenk bepinselten, bis dahin psoriasisfreien Patienten von Oscar Simon ${ }^{1}$ ) brach zuerst über jenem und von da ausstrahlend Psoriasis aus. Zu den chemischen würden nach der von Neißer wieder aufgenommenen v. Baerensprung schen Hypothese "embolischer Herde“ auch die ganz unerwiesenen Toxine der Psoriasis gehören. Schon Wertheim ${ }^{2}$ ), der, durch das angeblich häufigere Auftreten von Rezidiven an Armen und Beinen aufmerksam gemacht, Hunden Aufschwemmungen von Hefezellen injizierte, hatte die entstandenen, zum Teil über das Niveau der Haut hervortretenden Petechien, über welchen nach ihrer Rückbildung eine Abschilferung ihrer Epidermis eintrat, für Plaques gehalten. Hiergegegen - also gegen die ,Einschwemmung ihrer pathogenen Keime vom Blute aus" - sprechen meine direkten Experimente mit intravenösen Injektionen feinst zerriebener Psoriasisschüppchen an weißhaarigen Hunden.

Schon 1861 hatte ich ${ }^{3}$ ) auf denselben diagnostischen Irrtum von Tierärzten [Höring $\left.\left.{ }^{4}\right)\right]$ und neuerdings von Meurmann ${ }^{5}$ ) und Hafner ${ }^{6}$ ) und Tenholt ${ }^{7}$ ) bezüglich der Häufigkeit der Uebertragung der Psoriasis auf Kühe von Melkerinnen, seltener von Melkern hingewiesen.

Bekanntlich haben Lang (Wien) und A. Wolff (Straßburg), welche früher pilzähnliche Gebilde in der Epidermis beschrieben hatten, diese als fettige Ausscheidungen zurückgenommen.

Bezüglich der Häufigkeit "nervöser Einflüsse“, und zwar peripher oder zentraler mögen Polotebn off und Sirsky ${ }^{8}$ ) speziell bezüglich mikroskopischer Veränderungen im Rückenmark etwas übertrieben haben. Leloirs Stütze, Déjène, fand selbst derartige Veränderungen in den Hautverästelungen von Tabikern. Ferner sah ich bei einem Bruderpaar ländlicher Arbeiter, welche nackt länger als ein Jahr in demselben Bett geschlafen hatten, und deren älterer mit ausgebreiteter Psoriasis und Pityriasis versicolor behaftet war, nur letztere sich übertragen, erstere nicht.

W. Scholz' ${ }^{9}$ ) leitete aus der Regelmäßigkeit der Abheilung zweier Psoriasgürtel am Rücken, deren oberer zwischen den Spinae scapularum, der untere in der Lendengegend quer ausgebreitet war, durch subkutane Arseninjektionen selbst an entfernten Körpergegenden durch Aussaat einer bestimmten Penicilliumspezies seine "bioskopische Methode“ kleinster Arsenmengen in den Schuppen und Haaren ab.

Adrian und Pribram, ${ }^{10}$ ) Eger, ${ }^{11}$ ) Gerhard t, ${ }^{12}$ ) vor allen aber Besnier ${ }^{13}$ ) leiteten die mit Psorias koinzidierenden Gelenkerkrankungen von zentralen, durch die mikroskopischen Untersuchungen von Mario Oro ${ }^{14}$ ) neuerdings sichergestellten Veränderungen des Rückenmarks ab.

Von klinischen Beobachtungen, die für Uebertragbarkeit der Psoriasis $\mathrm{zu}$ sprechen scheinen, sind zunächst die von Unn ${ }^{15}$ ) und Niels en ${ }^{16}$ ) zu erwähnen: 1 . Eine psoriatische Amme tritt in eine psoriasisfreie Familie ein, und bald darauf erkranken drei Kinder an Psoriasis. Nielsens Fall betraf ein Kind und seine Mutter; letztere bekam aber die Psoriasis zum erstenmal erst längere Zeit nach der Tochter.

Aus einer Reihe von Beobachtungen, z. B. denen von Eger, ${ }^{17}$ ) ist zu ersehen, daß dem chronischen Gelenkrheumatismus häufig ein akuter vorausgegangen ist und daf im Verlaufe jenes nicht selten akute Exazerbationen sich einstellen. Ferner pflegt selbst bei langandauernden. schweren Gelenkleiden Psoriatischer das Herz intakt $z$ ubleiben. Auch Gerhardt ${ }^{18}$ ) meinte

1) Wutzdorff, Zur Aetiologie der Psoriasis und meine Erwiderung. Zeitschrift fur Dermatologie 1876 und 1877. - 2) Werthe i m1, Zur Aetiologieis der Psoriasis. Wiener medizinische Jahrbücher 1876. - 3) K $\mathrm{b}$ b ner, Ueber Sycosis und ihre Beziehungen zur Mycosis tonsurans. Virchows Archiv 186l. - 4) Höring zitert in meiner Arbeit über Sycosis und ihre Beziehungen zur Mycosis tonsurans. Virchows Archiv 1861. - 5) Meurman n, Deutsche medizinische Wochenschrift 1895. - 6) Hafner, Medizinische Korrespondenzblatter des Württembergischen ärztlichen Vereins $1856 .-7$ ) Tenhold, Korrespondenzblătter des allgemeinen ärztlichen Vereins von Thüringen 1888. - 8) Poloteb n of f und Girsky, (Russisch). Dermatologische Studien. Zweite Reihe fünftes Heft 1891. -

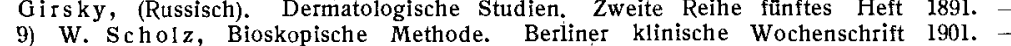
9) W. Scholz, Bioskopische Methode. Berliner klinische Wochenschrift 1901. -
10) Adrian und Pribram, Theorie des Wesens der Psoriasis. Deutsche medizinische 10) Adrian und Pribram, Theorie des Wesens der Psoriasis. Deutsche medizinische
Wochenschrift 1901. - $\rightarrow$ li) Eger, Ueber das Verhältnis der Schuppenflechte zu GelenkWochenschrift $1901 . \rightarrow$ li) Eger, Ueber das Verhältnis der Schuppenflechte zu Gelenk-
erkrankungen. Berliner kHinische Wochenschrift 1895 , No. 27. - 12) Gerh ardt, Ueber das Verhăltnis der Schuppenflechte zu Gelenkerkrankungen. Ibidem und IX. Kongre für innere Medizin 1896. - 13) Besnier in Bourdillons These: Contribution à la Pathologie et Thérapie de la Psoriasis. Paris 1896. - 14) Mario Oro, Contributo all Patologia o Therapia della Psoriasi. Annale internationale delle Sciluze mediche Napol 1903. - 15) U n na, Verhandlungen des allgemeinen internationalen Kongresses in Kopenhagen 1884. - 16) $\mathrm{Nie} / \mathrm{s}$ e $\mathrm{n}$, Verhandlungen des allgemeinen internationalen Kongresses in Kopenhagen 1884. - 17) Eger, Ueber die Verhältnisse der Schuppenflechte zu Gelenk erkrankungen. Berliner klinische Wochenschr. 1895, No. 27. - 18) Gerhardt, Ueber die Verhältnisse der Schuppenflechte zu Gelenkerkrankungen Ibidem 94 und X1V. Kongres fǜ nnere Medizin 1896. 
in drei Fällen von Gelenkerkrankungen bei Psoriasis, daß das bei den Prozessen zu Grunde Liegende in einer Nervenerkrankung zu suchen sei.

Vor allem aber ist die äußerst fleißige Arbeit von Martin Kutznitzky ${ }^{1}$ ) zu erwähnen: Bei einem sehr kräftigen Metzger, der sich mit einem Wiegemesser in den rechten Daumen bis auf den Knochen geschnitten hatte, kam fünf Wochen später, von dort auf dem Vorder- und Oberarm, Kopf, Achsel, Brust, Rücken, Flanke, Hinterbacke, Oberschenkel, streng halbseitig Psoriasis zum Vorschein.

Die Therapie ist, abgesehen von den altbekannten Topicis und Arsen, eine rein empirische geblieben: Alloxan (30\% iges Wasserstoff-Superoxyd) nach Sack und Neißer, und Asanol ein von den Harzsäuren befreites und fast geruchloses Teerpräparat - das fast geruchlose „Empyreum“ von Waelsch und Neißer. Die Therapie, speziell die Balneotherapie, des gesamten Hautorgans hat bisher trotz der besseren Kenntnis der Hautfunktionen keine Fortschritte gemacht: Das ehrliche Eingeständnis von Schultz (Kreuznach) ${ }^{2}$ ) über die „Fable convenue“ von der Heilkraft der Solbäder, welchem sich der Glaube an die der Schwefel- (Aachen, Nenndorf, Schinznach und besonders der Akratothermen (Leuk) anschließt, bezeugt ihre Nutzlosigkeit gegen Rezidive. Alle diese Faktoren sind bisher an Tausenden von Psoriasiskranken als schädlich nachgeprüft, die praktischen, speziell balneotherapeutischen Folgerungen aber bisher von niemandem gezogen worden.

Ich warne daher, weil ich davon die meisten Rezidive gesehen habe, vor kalten Fluß-, Sol-, vor allem aber vor Nordseebädern und empfehle nur häufige warme Wannenbäder, deren Wirkung allenfalls durch Zusatz schwacher $\left(1 \%{ }_{00}\right)$ Sodalösung oder Abreibung mit Schmierseife verstärkt werden kann. 